\title{
Evaluation of coupling losses in hollow-core photonic crystal fibres
}

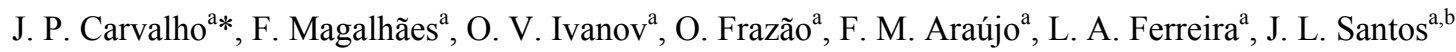 \\ ${ }^{a}$ INESC Porto - Institute for Systems and Computer Engineering of Porto, Porto, Portugal; \\ ${ }^{\mathrm{b}}$ Physics Department of Faculty of Science of University of Porto, Porto, Portugal \\ *email: joel.carvalho@inescporto.pt
}

\begin{abstract}
Hollow-core photonic crystal fibres have a high potential for gas sensing applications, since large light-gas interaction lengths can be effectively attained. Nevertheless, in order to enhance effective diffusion of gas into the fibre hollow-core, multi-coupling gaps are needed, which raise coupling loss issues that must be evaluated prior to the development of practical systems. In this communication we present a study on the coupling losses dependence on lateral and axial gap misalignment for single-mode fibre and two different types of hollow-core photonic crystal fibres. In addition, experimental results on the splicing of these fibres are also presented.
\end{abstract}

Keywords: hollow core photonic crystal fibre, coupling losses, splicing, gas sensing.

\section{INTRODUCTION}

Photonic crystal fibres (PCFs) [1,2] have generated a wave of excitement because of their promising properties that cannot be achieved with conventional optical fibres. In these fibres, the cladding is formed by a periodic pattern of micron-sized holes running along fibre length. The main feature of such periodic structures is that by varying the size and location of the holes, the fibres mode shape, nonlinearity, dispersion or birefringence can be tailored to reach values that are out-of-range for conventional single mode fibres (SMF). PCFs can be divided in two main groups: solid core fibres and hollow core $(\mathrm{H}(\mathrm{C})$ fibres [3]. There are also two mechanisms for transmitting light: index-guiding, present in both solid and hollow core PCF; and bandgap guiding, which appears only in HC-PCFs.

In the hollow core PCFs light is trapped in the core not by total internal reflection, but by a photonic bandgap in the cladding that acts like an insulator for light. The HC-PCF cladding is made with hundreds of periodically spaced air holes in a silica matrix, typically arranged in a honey combed-like pattern. Because the light guiding is no longer dependent on the core's effective index, it becomes possible to create fibres that guide light in an empty or gas-filled core. Thus HC-PCFs are finding an important niche of application in the gas-sensing area since large light-gas interaction lengths can be attained [4].

To practically exploit this feature it is still necessary to ensure proper diffusion of gas inside the fibre hollow-core. Several approaches can be envisaged to provide straight apertures through the cladding, creating multiple access points for the gas to reach the hollow-core. However, methods such as hole drilling often lead to asymmetrical transverse disruption of the honey combed structure resulting in intolerable losses. Multi-coupling gaps overcomes this limitation, being one of the most promising methods for the implementation of practical devices. Nevertheless, the optimum design of multiple-coupling gaps still requires a careful assessment of excess loss dependence on lateral and axial gap misalignment, since optical power needs to be kept below safe levels imposed in most hazard gas sensing application (e.g., methane).

Moreover, to be practically useful in remote gas detection systems, a PCF must also be connected to standard single mode fibre (SMF), which is still a rather delicate process. The splice losses between PCFs and SMFs have been studied in the last years by various groups [5-9]. Here a study of the splicing and coupling losses is performed for two different types of HC-PCFs: 19-cell and 7-cell HC-PCF. A brief modelling analysis and experimental measurements are presented, that allow multiple-coupling gaps loss assessment in these types of fibres.

\section{MODDELLING}

The coupling coefficient between two fibres (1 and 2) can be determined from their mode profiles (see Eq. (1)). If the fibres are separated by a distance $d$ (as depicted in Fig. 1), the mode field of one fibre has to propagate to the cut-end of the other fibre, as described in Eq. (2).

$$
C_{12}=\frac{\int E_{1} E_{2}^{*} d S}{\sqrt{\int\left|E_{1}\right|^{2} d S \int\left|E_{2}\right|^{2} d S}}
$$

$$
C_{12}=\frac{\int E_{1}(d) E_{2}^{*}(0) d S}{\sqrt{\int\left|E_{1}\right|^{2} d S \int\left|E_{2}\right|^{2} d S}}
$$




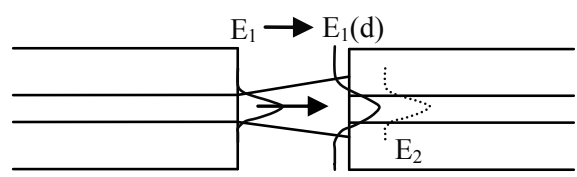

Figure 1 - Coupling between two fibres separated by distance $\mathrm{d}$.
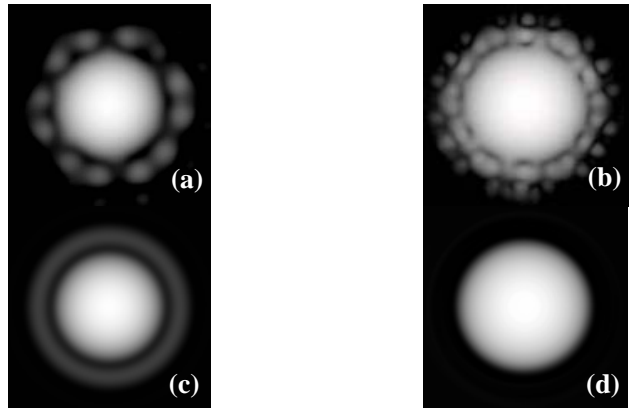

Figure 2 - (a) Mode of a 7-cell HC-PCF; (b) Mode of a 19-cell HC-PCF; (c) Radially averaged mode of a 7-cell HC-PCF; (d) Radially averaged mode of a 19-cell HC-PCF.

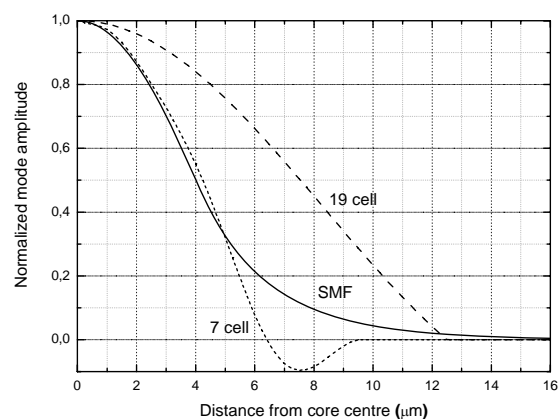

Figure 3 - Radially average of mode profiles for SMF, 7-cell HC-PCF, and 19-cell HC-PCF.
The mode field of an SMF can be easily computed. The mode fields of a 7-cell HC-PCF and a 19-cell HC-PCF are presented in figures 2-(a) and (b). The modes of HC-PCF are not radially symmetric, therefore to simplify our analysis we radially averaged field amplitudes of HC-PCF modes. The side lobes around the central spot of the 19-cell HC-PCF are small and their amplitudes have alternating signs. The light transmitted by these side lobes strongly diverge and will miss the second fibre core. Also, the side lobes of the first fibre are very probably misaligned with the side lobes of the mode of the second fibre.

Our estimation for the portion of light propagating in the side holes of a 19-cell HC-PCF is $1.8 \%$. Therefore, we assume that the light in the side lobes of the 19-cell HC-PCF is effectively lost and not coupled to the other fibre. The average mode profiles of the 7-cell HC-PCF, and the 19-cell HC-PCF are shown in figures 2-(c) and (d), and also in Fig. 3.

We used the mode profile of the left fibre of Fig. 1 (assuming it is centre-symmetrical) and simulated how it propagates in the free space between the two fibres by decomposing the beam into space Fourier components. The coupling coefficients for SMF $\rightarrow$ 7-cell HC-PCF, SMF $\rightarrow$ 19-cell HC-PCF, and 19-cell HC-PCF $\rightarrow$ 19-cell HC-PCF, were then calculated according to Eq. 2.

The decomposition of mode profiles of the SMF, the 7cell HC-PCF, and the 19-cell HC-PCF showed that more than $95 \%$ of the beam is in the zero-order Gaussian beam. The major difference between the fibres under analysis is then in the mode field diameter. Therefore, it is a good approximation to consider that the beams have Gaussian profiles.

The coupling coefficient between two Gaussian beams radiated from two fibres at distance $d$ can be found from Eq. 2 and is presented as follows:

$$
\left|C_{12}\right|^{2}=\frac{4}{2+\frac{z_{2}}{z_{1}}+\frac{z_{1}}{z_{2}}+\frac{d^{2}}{z_{1} z_{2}}},
$$

where $z_{i}=\pi w_{i}^{2} / \lambda$ and $w_{i}$ are the beam waists. Fig. 4 shows computed results from this equation.

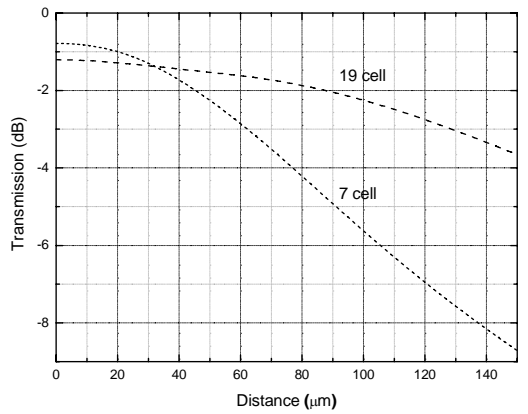

(a)

Figure $4-(\mathrm{a})$

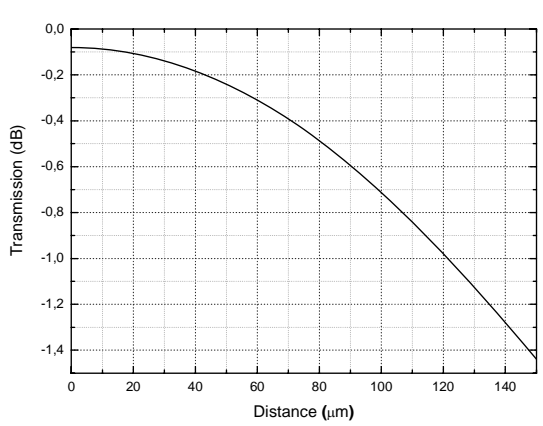

(b)

; (b) Coupling coefficients: 19-cell HC-PCF $\rightarrow$ 19-cell 


\section{EXPERIMENTAL RESULTS}

For evaluating gap coupling loss between a SMF and a HC-PCF, and also between two HC-PCFs, several measurements were made to evaluate the dependence on lateral and axial gap misalignment. A tuneable laser with $10 \mathrm{~mW}$ of maximum power was used as optical power source. The alignment between different fibres was achieved through a system with an axial step resolution of $5 \mu \mathrm{m}$ and a horizontal/vertical step resolution of $0.1 \mu \mathrm{m}$. The light detection was made through a large area detector for the $1.55 \mu \mathrm{m}$ wavelength region.

Fig. 5 shows the obtained results for axial displacement between SMF and HC-PCFs, and between two HC-PCFs of the same type. The excess loss values presented are referenced to minimal loss corresponding to direct fibre connection between the laser and the photodetector.

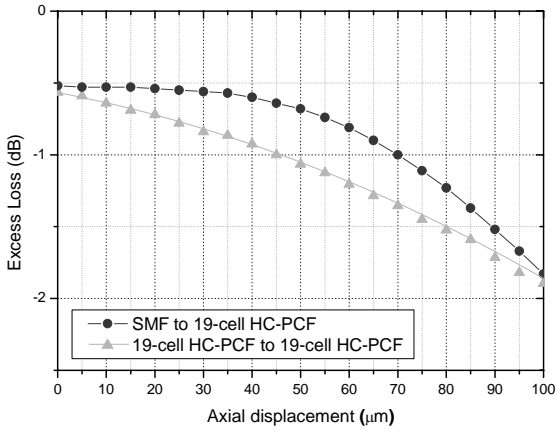

n.

(a) between a SMF and 19-cell HC-PCFs, and between two 19-cell HC-PCFs;

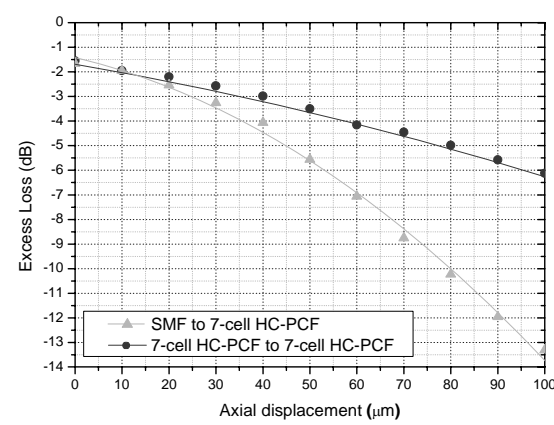

(b)

(b) between a SMF and 7-cell HC-PCF, and between two 7-cell HC-PCFs.

The obtained results show that for similar axial displacements, the 19-cell HC-PCF has much lower loss dependence than the 7-cell HC-PCF.

Fig. 6-(a) shows the obtained results for lateral displacement between SMF and HC-PCFs. Fig. 6-(b) shows the results for lateral displacement between two 19-cell HC-PCFs. During these measurements, the fibres were kept in close proximity.

The presented results confirm the previous ones tendency, the 19-cell HC-PCF loss coupling has much lower dependence on lateral displacement both with SMF and with another 19-cell HC-PCF. The coupling losses in the 7-cell HC-PCF have always a larger dependence either on lateral or axial gap misalignment. This behaviour was expected due to the higher mode field diameter of the 19-cell PCF when compared to both the SMF fibre and the 7-cell PCF which are quite similar. These results indicate that 19-cell HC-PCF is less susceptible to misalignments induced during multiplecoupling gaps implementation or during system operation due to environmental effects.

(a)

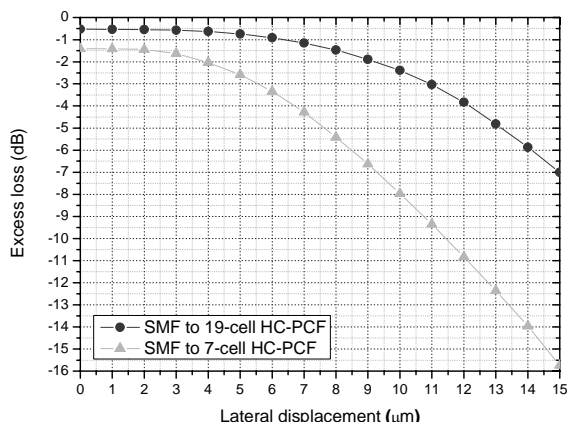

(b)

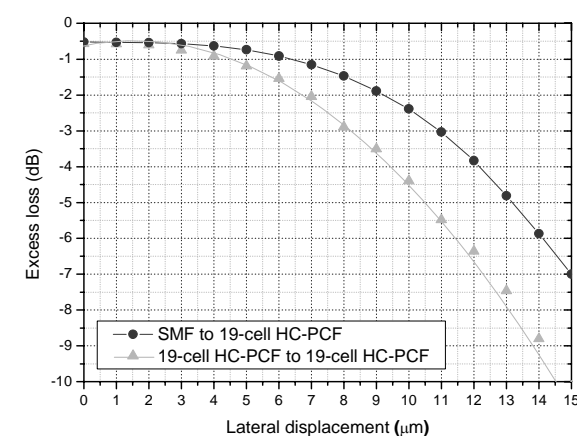

Figure 6 - Excess loss dependence on lateral displacement: (a) between a SMF and 19-cell HC-PCF and between a SMF and a 7-cell HC-PCF; (b) between a SMF and 19-cell HC-PCFs, and between two 19-cell HC-PCFs.

Furthermore, spectral measurements were performed for evaluating splicing losses at different conditions between SMF and 19-cell HC-PCF. Obtained results are presented in Fig. 7. From our experiments we concluded that for a arc current around $13.5 \mathrm{~mA}$ the ideal electric discharge time is around the $300-400 \mathrm{~ms}$, using a splicing technique previously reported [8]. 
The use of these splicing parameters allows reproducible splice losses to be attained. In Fig. 8 photographs of splices obtained under different conditions are presented. The physical shapes of the different splices do not significantly affect the splice losses between SMF and HC-PCFs. Nevertheless, even using optimum splice parameters the coupling efficiency is always lower when compared with straight butt-coupling.

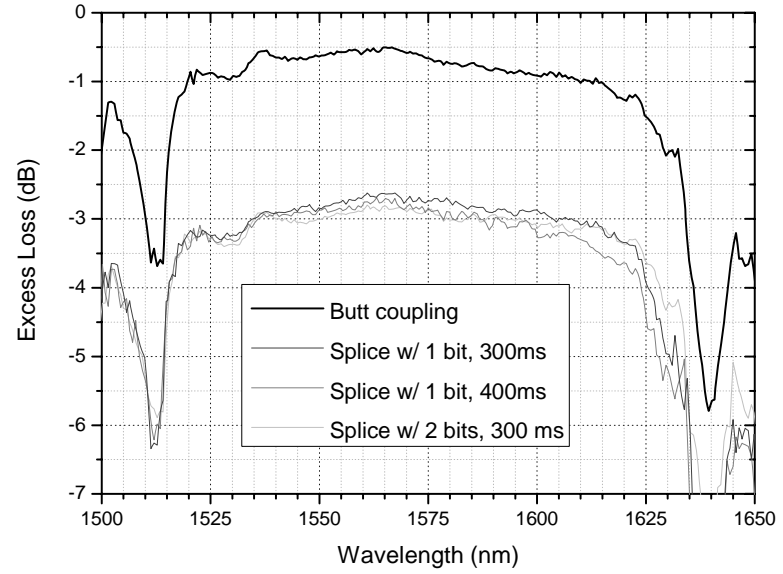

Figure 7 - Experimental results obtained for estimation of losses in light coupling between SMF and 19-cell HC-PCF in different cases.

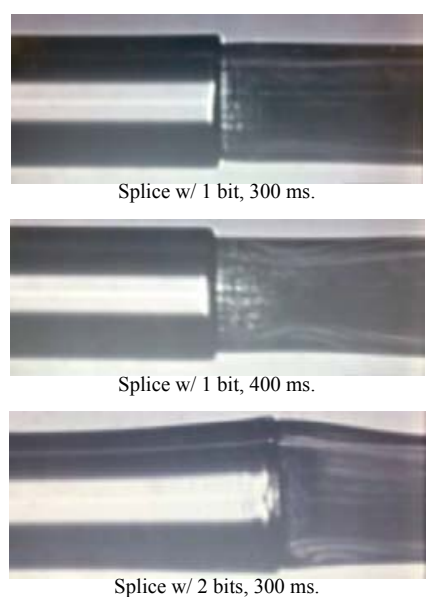

Figure 8 - Photographs of the different splices between SMF and 19-cell HC-PCF showed in Fig. 7.

\section{CONCLUSIONS}

In this work an analysis of the coupling losses dependence on axial and lateral displacement between SMF and HCPCF fibres was performed. It was found that 19-cell HC-PCF presents lower coupling losses than 7-cell HC-PCF. In light coupling between HC-PCFs of the same type we observed that loss dependence is higher for both axial and lateral misalignments. However, the obtained results are very encouraging towards the implementation of practical multiplecoupling gap based gas sensing systems.

A simple analysis on splice losses between 19-cell HC-PCF and SMF was also done, being the lowest insertion loss attainable $\sim 2 \mathrm{~dB}$.

\section{ACKNOWLEDGMENTS}

This work was developed in the framework of the European Project NextGenPCF, which is supported by IST in the 6th Framework R\&D Programme.

The authors acknowledge J. C. Knight from Department of Physics, University of Bath, for providing the hollow-core photonic crystal fibres used in the experiments.

\section{REFERENCES}

1. J. C. Knight, "Photonic crystal fibres", Nature, Vol. 424, No. 14 August, pp. 847-851 (2003)

2. P. Russel, "Photonic Crystal Fibers", Science, Vol. 299, No. 17 January, pp. 358-362 (2003)

3. C. M. Smith, N. Venkataraman, M. T. Gallagher, D. Müller, J. A. West, N. F. Borrelli, D. C. Allan and K. W. Koch, "Low-loss hollow-core silica/air photonic bandgap fibre", Nature, No. 7 August, Vol. 424, pp. 657-659 (2003)

4. Y. L. Hoo, W. Jin, C. H. L. Ho, D. N. Wang, S. C. Ruan, "Design and modelling of a photonic crystal fiber gas sensor", Applied Optics 42, 3509-3515 (2003)

5. J. H. Chong and M. K. Rao, "Development of a system for laser splicing photonic crystal fiber", Optics Express 11, 1365-1370 (2003)

6. B. Bourliaguet, C. Paré, F. Émond, A. Croteau, A. Proulx, and R. Vallée, "Microstructured fiber splicing", Optics Express 11, 3412-3417 (2003)

7. D. L. Bisbee, "Splicing silica fibers with an electric arc", Applied Optics 15, $796-798$ (1976)

8. J. T. Lizier, G. E. Town, "Splice losses in holey optical fibers", IEEE Photonics Technology Letters 13, 794-796 (2001)

9. O. Frazão, J. P. Carvalho and H. M. Salgado, "Low loss splice in a microstructured fibre using a conventional fusion splicing", Microwave and Optical Technology Letters, Vol. 46, No. 2, July 20, 172-174 (2005) 